All the terms in the product of these two series, after the second term, vanish. Putting the sum of the first two terms $=N=0$, we have $a_{0}-d\left(a_{0}+1\right)=0$. If we assume $a_{0}=-\frac{10}{9}$, then $d=10$, and the two factor series become

$$
\begin{aligned}
U & =-\frac{10}{9}-11 \frac{1}{9}-112 \frac{2}{9}-\cdots \\
U^{\prime} & =1-11+9+11-9-11+9+11-\cdots
\end{aligned}
$$

Since $U \cdot U^{\prime}=0$, we have $U^{p} \cdot U^{\prime p}=0$. As all the terms in $U$ are of the same sign, it is easily seen that $U^{p}$ is divergent for all positive integral values of $p . \quad U^{\prime}$ and $U^{\prime p}$ are also divergent.

$\S 8$. If we assume $t=2, s=1, c=-1, a_{0}=1, b_{0}=1$, $N=0$, then the condition that the sum of the product of $(5)$ and (6) shall vanish becomes $d^{2}+1=0$ and (letting $i=\sqrt{-1}$ ) the factor series thus obtained are the two complex divergent series

$$
\begin{aligned}
& 1+(1+i)+i+0+1+(i+1)+i+0+\cdots \\
& 1-(1+i)+(i-1)+(i+1)-(i-1)-(i+1)+\cdots
\end{aligned}
$$

Colorado College, Colorado Springis, April 12, 1902 .

\title{
THREE SETS OF GENERATIONAL RELATIONS DEFINING THE ABSTRACT SIMPLE GROUP OF ORDER 504.
}

BY PROFESSOR L. E. DICKSON.

(Read before the American Mathematical Society, October 25, 1902.)

1. Considerable interest attaches to the simple group of order 504. The existence of this simple group was discovered by Professor Cole.* This was one of the facts that lead Professor Moore $\dagger$ to his investigation of the linear fractional group in the general Galois field, resulting in the discovery of a new doubly infinite system of simple groups. 1893.

* "On a certain simple group," Mathematical Papers, Chicago Congress of + Buxletris, December, 1893 ; Mathematical Papers, Congress of 1893. 
The first set of generational relations follows readily from a general theorem quoted, with references, in $\S 2$. From it is derived, in $\S \S 5-9$, the second set of generational relations

$$
A^{7}=I, \quad B^{2}=I, \quad(A B)^{3}=I, \quad\left(A^{3} B A^{5} B A^{3} B\right)^{2}=I .
$$

The same method was followed by the writer * to determine the well-known generational relations for the simple linear fractional groups in the $G F[5]$ and $G F\left[2^{2}\right]$, each of order 60 ; and for that in the $G F[\bar{\tau}]$, of order 168. In a "Note on the simple group of order 504," Burnside † established the above second set by a direct analysis. Other derivations of this second set are due to R. Fricke \$ and to De Séguier. $\$$

The simplest of the three is the (new) third set

$$
C^{9}=I, \quad D^{2}=I, \quad(C D)^{3}=I, \quad\left(C^{3} D C^{5} D\right)^{2}=I .
$$

\section{First Set of Generational Relations, §§ 2-4.}

2. Theorem.\| The group of all linear fractional substitutions of determinant unity in the $G F\left[p^{n}\right]$ is simply isomorphic with the abstract group generated by the operators $T$ and $S_{\lambda}$ ( $\lambda$ running through the series of $p^{n}$ marks of the field), subject to the generational relations

$$
\begin{gathered}
T^{2}=I, \quad S_{0}=I, \quad S_{\lambda} S_{\mu}=S_{\lambda+\mu} \quad(\lambda, \mu \text { any marks }), \\
S_{\lambda} T S_{\mu} T S_{\frac{\lambda-1}{\lambda \mu-1}} T S_{-(\lambda \mu-1)} T S_{\frac{\mu-1}{\lambda \mu-1}} T=I
\end{gathered}
$$

( $\lambda$ and $\mu$ any marks such that $\lambda \mu \neq 1$ ).

3. For $p^{n}=2^{3}$, the group is a simple group of order 504 . I set the $G F\left[2^{3}\right]$ be defined by the irreducible congruence

$$
i^{3} \equiv i+1
$$

so that the marks of the field are

$$
0, \quad 1, \quad i, \quad i+1, \quad i^{2}, \quad i^{2}+1, \quad i^{2}+i, \quad i^{2}+i+1 .
$$

* Linear Groups, pp. 302-303.

+ Math. Annalen, vol. 52 (1899), pp. 174-176.

¥ Ibid., p. 335.

§Journal de Math., series 5, vol. 8 (1902), p. 267.

$\|$ Found, but not yet published, by Professor Moore. A different proof for finite fields is dne to the writer : Linear Groups, pp. 300-302. 
The relations $S_{\lambda} S_{\mu}=S_{\lambda+\mu}$ all follow from

(3) $S_{1}^{2}=S_{i}^{2}=S_{i+1}^{2}=S_{i^{2}}^{2}=S_{i^{2}+1}^{2}=S_{i^{2}+i}^{2}=S_{i^{2}+i+1}^{2}=I$,

(4) $S_{1^{\prime}} S_{i}=S_{i+1}, S_{1^{2}} S_{i^{2}}=S_{i^{2}+1}, S_{1^{1}} S_{i^{2}+i}=S_{i^{2}+i+1}, S_{i^{2}} S_{i}=S_{i^{2}+i^{2}}$

Taking the inverse of every member of (4) and applying (3), we get

$$
S_{i} S_{1}=S_{i+1}, \quad S_{i^{2}} S_{1}=S_{i^{2}+1}, \quad S_{i^{2}+i} S_{1}=S_{i^{2}+i+1}, \quad S_{i} S_{i^{2}}=S_{i^{2}+i^{*}}
$$

To show that $S_{i} S_{\mu}=S_{i+\mu}$ for every mark $\mu$, we note that

$$
\begin{aligned}
& S_{i} S_{i+1}=S_{i} \cdot S_{i} S_{1}=S_{1}, S_{i} S_{i^{2}+1}=S_{i} \cdot S_{i^{2}} S_{1}=S_{i^{2}+i} S_{1}=S_{i^{2}+i+1} \\
& S_{i} S_{i^{2}+i}=S_{i} \cdot S_{i} S_{i^{2}}=S_{i^{2}}, S_{i} S_{i^{2}+i+1}=S_{i} \cdot S_{i^{2}+i} S_{1}=S_{i^{2}} S_{1}=S_{i^{2}+1}
\end{aligned}
$$

Then $S_{i+1} S_{\mu}=S_{i+1+\mu}$ follows at once, since

$$
S_{i+1} S_{\mu}=S_{1} S_{i} S_{\mu}=S_{1} S_{i+\mu}^{\gamma}=S_{1+i+\mu}
$$

That $S_{\lambda} S_{\mu}=S_{\lambda+\mu}\left(\lambda=i^{2}, i^{2}+1, i^{2}+i\right.$, or $\left.i^{2}+i+1\right)$ follow quite similarly.

4. For $\lambda=0$ or 1 or for $\mu=0$ or 1 , relation (2) reduces to *

$$
\left(T S_{1}\right)^{3}=I \text {. }
$$

Among the remaining relations (2) occur the following six :

$$
\begin{array}{r}
S_{i} T S_{i} T S_{i^{2}+i} T S_{i^{2}+1} T S_{i^{2}+i} T=I, \\
S_{i^{2}} T S_{i^{2}} T S_{i} T S_{i^{2}+i+1} T S_{i} T=I, \\
S_{i+1} T S_{i+1} T S_{i^{2}+1} T S_{i^{2}} T S_{i^{2}+1} T=I, \\
S_{i^{2}+1} T S_{i^{2}+1} T S_{i^{2}+i+1} T S_{i^{2}+i} T S_{i^{2}+i+1} T=I, \\
S_{i^{2}+i} T S_{i^{2}+i} T S_{i^{2}} T S_{i+1} T S_{i^{2}} T=I, \\
S_{i^{2}+i+1}^{Y} T S_{i^{2}+i+1} T S_{i+1} T S_{i} T S_{i+1} T=I .
\end{array}
$$

From these follow all the relations (2) in which neither $\lambda$ nor $\mu$ is 0 or 1 . For, in (6)-(11), each subscript $i, i+1, i^{2}, i^{2}+1$, $i^{2}+i, i^{2}+i+1$ is followed immediately by all of the others, excepting its reciprocal, provided the first subscript be regarded

*Thus, for $\lambda=0,(2)$ becomes $T S_{\mu} T S_{1} T S_{1} T S_{-\mu+1} T=I$. Transforming both members by $T S_{\mu}$, we get $T S_{1} T S_{1} T S_{1}=I$. 
as following the last ; while from (2) results an equivalent relation by using for the initial $\lambda, \mu$ any two consecutive subscripts. We have therefore the following

Theonem.* The abstract simple group of order 504 is generated by the operators $T, S_{1}, S_{i}, S_{i^{2}+1}, \dot{S}_{i^{2}}, S_{i^{2+1}}, S_{i^{2}+i}, S_{i^{2}+i+1}$ subject to the generational relations $T^{2}=I$ and $(3),(4),(5),(6),(7)$, (8), (9), (10), (11).

Second Set of Generational Relations, §§5-9.

5. Transforming both members of (8) by $T S_{i+1}$, we get

$$
S_{i+1} T S_{i+1} T S_{i+1} T S_{i+1}=S_{i^{2}+i} T S_{i^{2}} T S_{i^{2}+i^{*}}
$$

Denoting each product by $P$, we form $P T P$ and get

$$
S_{i+1}\left(T S_{i+1}\right)^{7}=S_{i^{2}+i} T S_{i^{2}} T S_{i^{2}+i} T S_{i^{2+}+i} T S_{i^{2}} T S_{i^{2}+i^{*}}
$$

In view of (10), the second member equals

Hence

$$
S_{i^{2+i}} T S_{i^{2}} T \cdot T S_{i^{2}} T S_{i+1} \cdot S_{i^{2}+i}=S_{i+1} \text {. }
$$

$$
\left(T S_{i+1}\right)^{7}=I \text {. }
$$

Set $A \equiv T S_{i+1}, B \equiv S_{i}$. By (3), (4), (5), (13),

$$
A^{7}=I, B^{2}=I,(A B)^{3}=I \text {. }
$$

6. From (10) and (7), we obtain respectively

$$
\begin{aligned}
& S_{i^{2}} T S_{i^{2}}=S_{i+1} T S_{i+1} T S_{i} T S_{i+1} T S_{i+1}=A^{-2} B A^{2} \\
& S_{i^{2}+i+1}=T S_{i} T S_{i^{2}} T S_{i^{2}} T S_{i} T .
\end{aligned}
$$

* Note added November 10. The relations (8), (9), (10) may be omitted. Indeed, we may write $(6)$ thus :

$$
S_{i} T S_{i} T S_{i^{2}+i+1} S_{1} T S_{i^{2+1}+1} T S_{i^{2}+i_{i}} T=I .
$$

Replacing $T S_{i} T S_{i^{2}+i+1}$ by $S_{i^{2}} T S_{i^{2}} T S_{i} T$, as may be done by (7), and then $T S_{1} T$ by $S_{1} T S_{1}$, as may be done by (5), we obtain (10), with its subscripts permuted cyclically. Again, we may write $(6)$ thus :

$$
S_{i} T S_{i+1} S_{1} T S_{i^{2}+i} T S_{i^{2}+1} T S_{i^{2}+i} T=I .
$$

Replacing $S_{i} T S_{i+1}$ by $T S_{i+1} T S_{i^{2}+i+1} T S_{i^{2}+i+1} T$, in virtue of (11), and then $T S_{1} T$ by $S_{1} T S_{1}$, in virtue of (5), we obtain a relation immediately equivalent to (9). Finally, we may write (7) thus :

$$
S_{i^{2}} T S_{i^{2}} T S_{1} \cdot S_{i+1} T S_{i^{2}+i+1} T S_{i} T=I .
$$

Replacing $S_{i+1} T S_{i^{2}+i+1} T$ by $T S_{i} T S_{i+1} T S_{i^{2}+i+1}$, in virtue of (11), and then $T S_{1} T$ ' by $S_{1} T S_{1}$, we obtain (8). 
But $A B A^{-1}=T S_{i} T$. Hence

$$
S_{i^{2}+i+1}=A B A^{-3} B A^{3} B A^{-1},
$$

(16) $S_{i^{2}+1}=B \cdot A B A^{-3} B A^{3} B A^{-1}=A^{-1} B A^{3} B A^{3} B A^{-1}$,

since $B A B=A^{-1} B A^{-1}$ by (14). Since $S_{i^{2}+1}$ is of period 2,

$$
\left(A^{3} B A^{5} B A^{3} B\right)^{2}=I \text {. }
$$

7. We next express all the generators of the group in terms of $A$ and $B$. Transforming (8) by $T S_{i^{2}+1}$, we get

$$
S_{i^{2}+1} A^{2} T S_{1^{2}} S_{i^{2}} T S_{i^{2}} T=I \text {. }
$$

Replacing $S_{i^{2}+1}$ and $S_{i^{2}} T S_{i^{2}}$ by their values and $T S_{1}$ by $A B$,

$$
T=A^{-1} B A^{3} B A^{3} B A^{2} B A^{5} B A^{2} \text {. }
$$

Since $B A^{2} B=A^{-1} B A^{-2} B A^{-1}$ by (14), we get

$$
\begin{aligned}
T & =A^{-1} B A^{3} B A^{2} B A^{-2} B A^{4} B A^{2} \\
& =A^{-1} B A^{2} B A^{-2} B A^{-3} B A^{4} B A^{2} .
\end{aligned}
$$

Replacing $B A^{-3} B A^{4} B A^{2}$, of period 2 by (17), by its inverse, we get

$$
T=A^{-1} B A^{2} B A^{3} B A^{3} B A^{3} B .
$$

8. The $S_{\mu}$ are given by (15), (16) and the relations $S_{1}=T A B, S_{i+1}=T^{-1} A, S_{i^{2}+i}=T A B S_{i^{2}+i+1}, S_{i^{2}}=S_{i^{2}+i} B$.

Some of these relations may be simplified. Thus,

$$
\begin{array}{rlr}
S_{i^{2}+i} & =A^{-1} B A^{2} B A^{3} B A^{3} B A^{3} B \cdot A B \cdot A B A^{-3} B A^{3} B A^{-1} \\
& =A^{-1} B A^{2} B A^{3} B A^{3} B A^{-1} B A^{3} B A^{-1} \quad[\text { by }(14)] \\
& =A^{-1} B A^{2} B A^{3} B A^{4} B A^{4} B A^{-1} & {\left[B A^{-1} B=A B A\right]} \\
& =A^{-2} B A^{-2} B A^{2} B A^{4} B A^{4} B A^{-1} & {\left[B A^{2} B=A^{-1} B A^{-2} B A^{-1}\right]} \\
& =A^{-2} B A^{-2} B \cdot B A^{3} B A^{3} B A^{5} \cdot A^{-1} & {[\text { by }(17)]} \\
& =A^{-2} B A B A^{3} B A^{4} & \\
& =A^{-3} B A^{2} B A^{-3} &
\end{array}
$$


1903.] THE SIMPLE GROUP OF ORDER 504.

Taking the inverse for $S_{i^{2+} i}$, we have the relations

$$
\left\{\begin{aligned}
S_{i} & =B, S_{1}=T A B, & S_{i^{2}+i} & =A^{3} B A^{5} B A^{3}, \\
S_{i+1} & =T^{-1} A, & S_{i^{2}} & =A^{3} B A^{5} B A^{3} B .
\end{aligned}\right.
$$

9. Theonem. The simple group of order 504 is generated by two operators $A$ and $B$ subject to the generational relations (14) and (17).

It remains to show that the operators defined by (15), (16), (18) and (19) satisfy the relations $T^{2}=I,(3)-(11)$, in view of relations (14) and (17) alone. We derive at once the relations

$$
\begin{aligned}
S_{i}^{2} & =I, S_{i^{2}+i+1}^{2}=I, S_{i^{2}+1}^{2}=I, S_{i^{2}}^{2}=I, S_{i^{2}} S_{i}=S_{i^{2}+i}, \\
S_{i^{2}+i}^{2} & =A^{3} B A^{5} B A^{-1} B \cdot A^{5} B A^{3} \\
& =A^{3} B A^{-1} B A^{-1} B A^{3}=A^{7}=I .
\end{aligned}
$$

Since $B A^{2} B=A^{-1} B A^{-2} B A^{-1}$ by (14), we get

$$
\begin{aligned}
T^{2} & =\left(A^{5} B A^{5} B A^{2} B A^{3} B A^{3} B\right)^{2} \\
& =A^{5} B A^{5} B A^{2} \cdot A^{2} B A^{4} B A^{4} \cdot A^{5} B A^{2} B A^{3} B A^{3} B \\
& =A^{5} B A^{5} \cdot B A^{4} B A^{4} B A^{2} B \cdot A^{2} B A^{3} B A^{3} B \\
& =A^{5} B A^{5} \cdot A^{5} B A^{3} B A^{3} \cdot A^{2} B A^{3} B A^{3} B \\
& =\left(A^{5} B A^{3} B A^{3} B\right)^{2}=I
\end{aligned}
$$

That $S_{i+1} \equiv T^{-1} A$ has period 2 is shown as follows :

$$
A T A T=B A^{2} B A^{3} B A^{3} B A^{5} B A^{3} B A^{3} B A^{3} B
$$

$$
=B A^{2} \cdot A^{2} B A^{4} B A^{4} \cdot A^{3} B A^{3} B A^{3} B=I
$$

[by (17)].

We may show that $S_{1}^{2}=I$ by indicating the square of

$$
T^{-1} A B=B A^{4} B A^{4} B A^{4} B A^{5} B A^{2} B
$$

and replacing $B A^{-1} B$ by $A B A$ six times in succession. We have therefore derived all the relations (3) and $T^{2}=I$. Next,

$$
\begin{gathered}
S_{1} S_{i}=T A B \cdot B=T^{-1} A=S_{i+1} ; \\
S_{1}=T A B=A^{-1} B A^{3} B A^{3} B A^{2} B A^{5} B A^{3} B=S_{i^{2}+1} S_{i^{2}},
\end{gathered}
$$


upon employing for $T$ formula (18') which follows from (18), (17), (14). Hence $S_{1^{2}} S_{i^{2}}=S_{i^{2}+1}$. Again,

$$
S_{1} S_{i^{2}+i}=S_{1} S_{i^{2}} S_{i}=S_{i^{2}+1^{2}} S_{i}=\left(S_{i^{2}} S_{i^{2}+1}\right)^{-1}=S_{i^{2}+i+1}^{-1}=S_{i^{2}+i+1} .
$$

Hence relations (4) have all been derived.

It remains to derive relations (5)-(11). From what precedes, we may make use of relations (1). Since $T S_{1}=A B$, (5) follows from (14). We next verify that

$$
S_{i^{2}} T S_{i^{2}}=A^{-2} B A^{2} .
$$

Employing $S_{i^{2}}=S_{i^{2}}^{-1}=B A^{4} B A^{2} B A^{4}$ and the value of $T$ from (18), we obtain as the condition for (21),

$$
A^{2} B A^{4} B A^{2} B A^{3} B A^{2} B A^{3} B A^{5} B A^{2} B=I .
$$

Replacing the first two products $B A^{2} B$ by $A^{-1} B A^{-2} B A^{-1}$, we get

$$
A^{2} B A^{3} B A^{-2} B A B A^{-2} B A^{2} B A^{5} B A^{2} B=I .
$$

Replacing $B A B$ by $A^{-1} B A^{-1}$ and then $B A^{4} B A^{4} B A^{2}$ by its inverse, in view of (17), we get

$$
A^{2} B A B A^{3} B A B A^{2} B=I,
$$

a relation following readily from (14). As in $\S 6,(10)$ and (7) follow from (21), (15), (16), and

$$
A B A^{-1}=A S_{i+1} S_{i} S_{i+1} A^{-1}=T S_{i} T .
$$

From $\left(18^{\prime}\right)$ and $(21)$ follows $(8)$ as in $\$ 7$. Note that (10) also follows from (8), and $A^{7}=I$, as in $\S 5$.

Employing (22), relation (6) may be written

$$
B \cdot A B A^{-1} \cdot S_{i^{2}+i} \cdot T S_{1} \cdot S_{i^{2}} T S_{i^{2}} \cdot S_{i} T=I .
$$

Applying (14), (19) and (21), the relation becomes

$$
A^{-1} B A^{-2} \cdot A^{3} B A^{5} B A^{3} \cdot A B \cdot A^{-2} B A^{2} \cdot B T=I .
$$

By (14) we may replace $B A^{5} B$ and $B A^{-2} B$ by $A B A^{2} B A$ :

$$
A^{-1} B A \cdot A B A^{2} B A \cdot A^{4} \cdot A B A^{2} B A \cdot A^{2} B T=I .
$$

Replacing $B A^{6} B$ by $A B A$, we get, by (18),

$$
A^{-1} B A^{2} B A^{3} B A^{3} B A^{3} B T=T^{2}=I \text {. }
$$


1903.] THE SIMPLE GROUP OF ORDER 504.

Transforming (11) by $T$, we get

$$
T S_{i+1} \cdot S_{i^{2}} T S_{i^{2}} \cdot S_{i+1} T \cdot S_{i+1} T \cdot S_{i} \cdot T S_{i+1}=I .
$$

Setting $T S_{i+1}=A$ and $S_{i}=B$, and applying (21), it becomes an identity.

To verify (9), we write it in the form

$$
S_{i^{2}+1} \cdot T S_{1} \cdot S_{i^{2}} T S_{i^{2}} \cdot S_{i+1} T \cdot S_{i} \cdot S_{i^{2}} T S_{i^{2}} \cdot S_{i+1} T=I .
$$

Applying (16), (21), $T S_{1}=A B, S_{i+1} T=A^{-1}, S_{i}=B$, it becomes

$$
A^{-1} B A^{3} B A B A B A^{-2} B A=I,
$$

which is an identity since $B A B A B=A^{-1}$ by (14).

Third Set of Generational Relations, §§10-12.

10. Consider the two operators

$$
C=S_{i} T, \quad D=S_{i+1} .
$$

From (6) and (8) we obtain respectively

$$
\left(S_{i} T\right)^{4}=S_{i^{2}} T S_{i^{2}+1} T S_{i^{2}} T, \quad T S_{i^{2}+1} T S_{i^{2}} T=S_{i+1} T S_{i+1} T S_{i^{2}+1} .
$$

Hence, applying also (11), we derive

$$
\left(S_{i} T\right)^{9}=\left(S_{i^{2}+i+1} T S_{i+1} T S_{i^{2}+1}\right)^{2} S_{i} T=I .
$$

Also, $D C \equiv S_{1} T$ is of period 3 by (5). Hence

$$
D^{2}=I, C^{9}=I,(D C)^{3}=I .
$$

11. Noting that $C^{-1} D C=T S_{i+1} T$, we may write (11) thus :

$$
\begin{aligned}
& S_{i^{2+i+1}} T S_{i^{2}+i} \cdot D C \cdot D \cdot C^{-1} \cdot C^{-1} D C=I, \\
& S_{i^{2+i+1}} T S_{i^{2}+i}=C^{-1} D C^{2} D C^{-1} D=C^{-1} D C^{3} D C .
\end{aligned}
$$

Multiplying the latter on the left by $S_{i+1}$ and on the right by $S_{i} T$, we get

$$
S_{i^{2}} T S_{i^{2}} T=D C^{-1} D C^{3} D C^{2}=C D C^{4} D C^{2} .
$$

Applying this result in (7), we get

$$
S_{i^{2}+i+1} T=C^{6} D C^{5} D C^{7} .
$$

We now obtain the formulæ*

* It may be shown that $S_{i+i+1}^{2}=D C^{7} D C^{3} D C^{7}$. 


$$
\begin{aligned}
& S_{i^{2}+i}=S_{i^{2}+i+1} T \cdot T S_{1}=C^{6} D C^{5} D C^{6} D \\
& S_{i^{2}+1}=S_{i^{2}+i} S_{i+1}=C^{6} D C^{5} D C^{6} \\
& S_{i^{2}} T=S_{i^{2}+i}^{-1} S_{i} T=D C^{3} D C^{4} D C^{4} .
\end{aligned}
$$

Since $S_{i^{2}+1}$ is of period 2, it follows from (25) that

$$
\left(D C^{5} D C^{3}\right)^{2}=I
$$

We may write (6) in the form

$$
C^{2} \cdot S_{i^{2}} T \cdot T S_{i} \cdot T S_{i^{2}} \cdot S_{1} T \cdot S_{i^{2}+i} \cdot T=I .
$$

Applying (26) twice and (24) once, this relation gives

$$
C^{2} D C^{3} D C^{4} D C^{-1} D C^{5} D C^{4} D C^{5} D C^{6} D=T .
$$

Replacing $D C^{-1} D$ by $C D C$ in view of (23) and then $C^{3} D C^{5} D$ by $D C^{t} D C^{6}$, in view of (27), we get

$$
T=C^{6} D C^{3} D C^{4} D C^{5} D C^{6} D \text {. }
$$

From the definitions of $A$ and $B$ at the end of $\S 5$, we get

$$
\begin{aligned}
& A=T D=C^{6} D C^{3} D C^{4} D C^{5} D C^{6}, \\
& B=C T^{-1}=C D C^{3} D C^{4} D C^{5} D C^{6} D C^{3} .
\end{aligned}
$$

12. TheOREM. The simple group of order 504 is generated by two operators $C$ and $D$, subject to the generational relations (23) and (28).

In view of the theorem of $\S 9$, it suffices to show that, if $A$ and $B$ are defined by (29) and (30), relations (14) and (17) follow from relations (23) and (27). First,

$$
B A=C T^{-1} \cdot T D=C D,
$$

so that $B A$ is of period 3 in view of (23). To show that $B^{2}=I$, we replace $D C^{6} D C^{4}$ by its inverse $C^{5} D C^{3} D$ in the indicated square of $B$ and get

$$
B^{2}=C D C^{3} D C^{4} \cdot D C D \cdot C^{6} D C^{4} D C^{5} D C^{6} D C^{3} \text {. }
$$

Replacing $D C D$ by $C^{-1} D C^{-1}$ and, in the result, $D C^{3} D C^{5}$ by $C^{4} D C^{6} D$, we get

$$
B^{2}=C D C^{7} D C D C^{5} D C^{6} D C^{3} .
$$

Making similar replacements, we get $B^{2}=C^{6} D C^{9} D C^{3}=I$. 
In the indicated product $A A$, we replace $D C^{5} D C^{3}$ by $C^{6} D C^{4} D$, then $D C D$ by $C^{-1} D C^{-1}$, then $C^{6} D C^{4} D$ by $D C^{5} D C^{3}$, then $D C^{-1} D$ by $C D C$ twice, and get

$$
A^{2}=C^{7} D C^{8} \text {. }
$$

It follows at once that

$$
A^{6}=C^{7} D C^{6} D C^{6} D C^{8} \text {. }
$$

It will equal the expression for $A^{-1}$ given by (29) if

$$
C^{4} D C^{6} D C^{6} D C^{5}=D C^{4} D C^{5} D C^{6} D \text {. }
$$

Replacing $C^{4} D C^{6} D$ by $D C^{3} D C^{5}$, the condition becomes

$$
I=C^{4} D C^{7} D C D C^{5} D C^{6} D .
$$

Replacing $D C D$ by $C^{-1} D C$ and then $C^{6} D C^{4} D$ by $D C^{5} D C^{3}$, the condition reduces to an identity in view of $D^{2}=I, C^{9}=I$.

Finally, to derive (17), we note that

$$
B A^{3} B A^{5} B A^{3}=B A \cdot A^{2} \cdot B A \cdot A^{4} \cdot B A \cdot A^{2},
$$

and substitute the values of $B A$ and $A^{2}$ given by (31) and (32). The result $C D C^{5} D C^{4} D C^{8}$ is evidently of period 2 by (23).

Generators of the Linear Fractional Group of Order 504.

13. For the first set of generational relations, we may take

$$
T: \quad z^{\prime}=\frac{1}{z} ; \quad S_{\mu}: \quad z^{\prime}=z+\mu .
$$

Hence, for the second set, we may take

$$
A: \quad z^{\prime}=\frac{1}{z}+i+1 ; \quad B: \quad z^{\prime}=z+i .
$$

For this second set, Burnside employs

$$
A^{\prime}: \quad z^{\prime}=i z ; \quad B^{\prime}: \quad z^{\prime}=\frac{z+i^{2}}{i^{2} z+1} .
$$

We readily obtain the relations

$$
\begin{aligned}
& A^{\prime}=T S_{i+1} T S_{i} T S_{i} T S_{i} T S_{i+1}=S_{i^{2}+i+1} T S_{i^{2}+i} T S_{i+1} T S_{i+1}, \\
& B^{\prime}=S_{i+1} T S_{i+1} T S_{i^{2}+i+1}^{\prime} T S_{i^{2}+i} .
\end{aligned}
$$

For the third set, we may evidently take

$$
C: \quad z^{\prime}=1 \div(z+i) ; \quad D: \quad z^{\prime}=z+i+1 .
$$


Note that $S_{i^{2}+1} T$ is of period 7 ; while

$$
B A^{3}: \quad z^{\prime}=\frac{z+i+1}{z}
$$

is of period 9 ; also, as a check on (27), that

$$
D C^{5} D C^{3}: \quad z^{\prime}=\frac{z+i^{2}}{i z+1} .
$$

The University of Chicago,

October 19, 1902.

\section{GENERATIONAL RELATIONS DEFINING THE ABSTRACT SIMPLE GROUP OF ORDER 660.}

BY PROFESSOR L. E. DICKSON.

(Read before the American Mathematical Society, October 25, 1902.)

THE abstract group of order 660 , simply isomorphic to the group of linear fractional substitutions of determinant unity taken modulo 11, may be generated by two operators $S$ and $T$ subject to the following relations: ${ }^{*}$

$$
S^{11}=I, \quad T^{2}=I, \quad(S T)^{3}=I,
$$

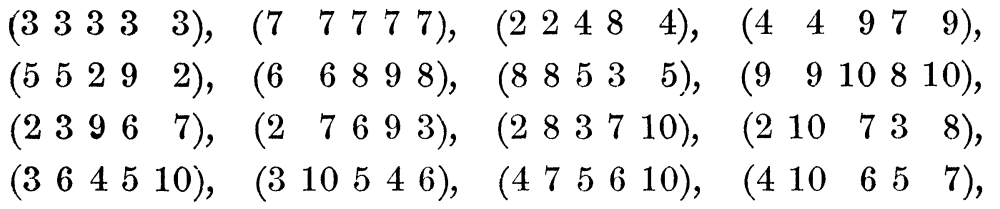
where the symbol $(a b c d e)$ denotes the relation

$$
S^{a} T S^{b} T S^{c} T S^{d} T S^{e} T=I .
$$

These relations are very redundant. We proceed to reduce

* For a proof of the general theorem due to Moore, see Dickson, Linear Groups, \& 278, Corollary. When the field is of order a prime $p$, we may set $S_{1}=S, S_{t}=S^{t}$. By $\xi 279$, we need retain only the 16 relations $(a, b, c, d, e)$ given above, since the others follow by a cyclic permutation of $a, b, c, d, e$. It may be shown that $S^{2} T, S^{3} T, S^{4} T, S^{5} T, S^{6} T, S^{7} T, S^{8} T, S^{9} T$ have the respective periods $12,5,5,6,6,5,5,12$, results not used here. 